\title{
Prostaglandins and leprosy. A role for aspirin?
}

\author{
P KLENERMAN \\ Royal United Hospital, Bath BA1 3NG
}

\section{Accepted for publication 21 October 1988}

Summary Prostaglandins not only have a role in inflammation, but may also be involved as mediators in the immune response. Drugs which affect prostaglandin synthesis may therefore be potential tools with which to modulate disturbed immunity. These possibilities are discussed with ref erence to immunity in leprosy, and in particular reversal reactions.

\section{Introduction}

All clinicians are aware of the role of prostaglandins as inflammatory mediators; non-steroidal antiinflammatory agents are crucial drugs in every formulary. Now, too, we are getting used to the idea of aspirin as a powerf ul antiplatelet agent, with significant effects on mortality from coronary heart disease and stroke. However, there may yet be another role for these drugs, for as the arachidonic acid metabolites are ubiquitous and multifaceted, so their inhibition may have widespread effects. It has been known for over 15 years that prostaglandins (PGs) may modulate the immune responsealthough how important this role is, is not yet clear. In this essay I would like to examine the potential effects of PGs on one disease, leprosy, and suggest that aspirin and its fellows may yet have a part to play in its therapy.

\section{Prostaglandin synthesis}

PGs are derived from arachidonic acid and its analogues, via an oxidative process. Arachidonic acid is present in the membranes of all cells, normally bound to phospholipid. The first step in PG synthesis therefore is release from the cell membrane through the action of the enzyme phospholipase A2. This enzyme is inhibited by steroids, which therefore present the synthesis of all arachidonic acid metabolites. ${ }^{1}$

Arachidonic acid available within the cell may then go down two pathways, the lipoxygenase pathway, to form leukotrienes, and the cyclo-oxygenase pathway. Action of the latter enzyme creates an intermediate $\mathrm{PGG}_{2}$ from which three main groups are derived, prostacyclin, thromboxane and the PGE and PGF series. Aspirin, indomethacin and the non-steroidal anti-inflammatory agents (NSAIA's) act on cyclo-oxygenase to inhibit the production of all these metabolites. They are thought to have little action on lipoxygenase synthesis (perhaps even causing an increase in flux through this pathway as a 'diversion' for-arachidonic acid), although recently it has been shown that there may be some inhibition in vitro. ${ }^{2}$ 
The biological functions of the PGs are numerous and beyond the scope of this essay. Prostacyclin and thromboxane have opposing eff ects on platelet aggregation and vasoconstriction, the former acting as an antagonist. PGs of the $\mathrm{E}$ and $\mathrm{F}$ series are pro-inflammatory agents, causing local vasodilatation, increased vascular permeability, and potentiating the action of histamine and bradykinin in causing pain and accumulating oedema. ${ }^{1}$ They are implicated in a number of other body systems, for example as endogenous pyrogen within the hypothalamus, and in renal homeostasis-however, in this essay, it is their involvement in immune regulation which is to be considered.

\section{Prostaglandins in normal immunity}

\section{IN VITRO STUDIES}

The first report directly suggesting that PGs may act as immunoregulatory agents came in 1971, when Smith and his colleagues noted that $\mathrm{PGE}_{2}$ inhibited incorporation of radiolabelled metabolites into human lymphocytes stimulated by mitogens. These results were then followed by those of a number of other investigators who showed that PGE could suppress in vitro markers of lymphocyte activity, e.g. antibody and lymphokine secretion. ${ }^{3}$ However, these results do not prove that PGE is an endogenous mediator of immunosuppression; any cell poison would have the same effect. The following observations argue in its favour:

1 Prostaglandins are produced in vitro in the proliferative response to mitogens.

2 Inhibition of PG synthesis by indomethacin causes a reduction in PG concentration and a rise in in vitro immune reponsiveness.

3 Addition of PG to these systems negates the effect of indomethacin. Importantly this effect is seen at physiological concentrations $\left(10^{-8}\right.$ to $\left.10^{-6} \mathrm{M}\right)$.

One study ${ }^{3}$ further narrowed down this role as an immunosuppressant to PGs of the E series. Physiological concentrations of these agents inhibit not only the lymphocyte proliferative response but also $\mathrm{E}$ rosetting, cytotoxic $\mathrm{T}$ cell and $\mathrm{NK}$ cell activity. There is evidence, however, that $\mathrm{PGE}_{1}$ may act as an immunostimulant in certain in vitro systems, ${ }^{4}$ e.g. response to BCG. Thus while the overwhelming balance of evidence is in favour of a suppressive role in most in vitro systems, this experiment reminds us that it is dangerous to extrapolate from one such system to the next, let alone to the whole animal. A related point is that the action of prostaglandins and antiprostaglandins in these studies is commonly restricted to a narrow time window at the beginning of the reaction, ${ }^{3}$ with minimal effects beyond $24-48 \mathrm{~h}$.

\section{IN VIVO STUDIES}

PGs may be present and active in the test-tube, but does this have any relevance to the whole animal? There have been few in vivo studies of PGs in the normal human immune response, although the continuous uncontrolled experiment with the thousands of individuals already taking antiprostaglandin agents would suggest that such effects are likely to be small. This does not mean, however, that they are either (a) insignificant, or (b) irrelevant to the disease state where there may already be a disorder of immune regulation.

There have been a number of experiments in mice which demonstrate enhanced antibody response to antigen challenge in vivo. ${ }^{3}$ Indeed, as long ago as 1968 , Robinson and colleagues demonstrated that mice pretreated with indomethacin had an enhanced ability to resist infections. However, perhaps most relevant of these animal experiments was the observation of one study ${ }^{5}$ that simultaneous oral administration of indomethacin resulted in a marked increase in skin thickness of the delayed type hypersensitivity (DTH) response to intradermal Mycobacterium tuberculosis. This 
startling result emphasizes just how carefully we must tread in this field, for most clinicians, trained with the idea of indomethacin as an anti-inflammatory agent, would have predicted exactly the opposite effect.

In normal humans, there has been no observed equivalent effect of indomethacin on skin test responses to mumps, candida and trychophyton. ${ }^{6}$ However, responses to an Influenza A vaccine were enhanced by pretreatment with indomethacin in normal volunteers. This only occurred in strains to which the individual had been previously sensitized, suggesting that PG synthetase inhibitors may act to stimulate the secondary rather than the primary repsonse. ${ }^{13}$

\section{How might prostaglandins act?}

While it is clear from the above experiments that PGs may have effects, whether major or minor, on in vitro and in vivo measures of immunity, there are a number of issues raised, perhaps the most important of which is how they might act.

The major PG producing cell amongst the mononuclear class is glass-adherent, and it is generally thought that the most important of these is the macrophage. ${ }^{7}$ This, by secreting PGE, may either directly suppress $\mathrm{T}$ cells or activate a non-glass adherent $\mathrm{T}$ suppressor subset (which does not act through prostaglandins). The exact mechanism involved in this immunosuppression is not clear, but is thought to involve both reduced IL2 secretion and IL2 responsiveness. ${ }^{8}$ PGs exert their effect through cyclic AMP within the cell ${ }^{9}$ and it is of note that indomethacin may have effects on levels of this metabolite directly via phosphodiesterase inhibition and may also act on cyclic AMPdependent protein kinase. In other words, indomethacin may have immunomodulatory effects independently of PG inhibition and this should be borne in mind in interpretation of in vitro and in vivo work.

Another complicating issue in understanding PG action is that of the effects of leukotrienes on the immune response. There is in vitro evidence that leukotrienes, notably $\mathrm{LTB}_{4}$, have antagonistic effects to PGs in immunity: lipoxygenase products stimulate mitogen-induced proliferation, which is inhibited by lipoxygenase pathway inhibitors. One effect of PG synthetase inhibitors may be, therefore, to channel more arachidonic acid into the lipoxygenase pathway, as has been proposed in aspirin-induced asthma. ${ }^{1}$

This mechanistic approach takes us away from the problem of the biological function of PGs in immunity. While we are used to the idea of positive feedback in the inflammatory response, if tissue damage is to be limited there must also be negative feedback between the inflammatory and immune cells. Within the immune system, $T$ suppressor cells and idiotypic networks have been proposed to fill such a role. Perhaps PGs, while enhancing the local inflammatory response act to damp the escalation of the developing immune reaction, by feedback inhibition. If this model is true, two predictions can be made. Firstly, we will only be able to fully understand the role of PGs if we consider the immune and inflammatory responses together, not in isolation. Secondly, there may be disease states in which over- or under-damping occurs, leading to hypo- or hyper-responsiveness.

\section{Prostaglandins and abnormal immunity}

There is evidence that PGs may play a part in various disease states with disordered immunity. In patients with Hodgkin's Disease, for example, where there is reduced cell-mediated immunity, T suppressor cells are found to produce four times the normal quantity of PGE. In vitro, this suppressive effect can be overcome with indomethacin. ${ }^{11}$ In tumour-bearing mice, tumour-induced immunosuppression may be reversed with indomethacin both in vitro and in vivo. Indomethacin not only reduces the rate of tumour growth, but also enhances the therapeutic effect of BCG. ${ }^{12}$ 
Perhaps the most fascinating experiment in this area has been that concerning the effects of indomethacin on 2 patients with common variable immunodeficiency, a disease associated with abnormal $\mathrm{T}$ suppressor function. ${ }^{13}$ These patients were previously anergic to skin testing with antigens including PPD (purified protein derivative from TB), but showed a return to skin test responsiveness and restoration of in vitro markers of cellular immunity upon drug therapy (disappearing on stopping the drug). This result, although on a small scale, suggests that such immunomodulation may be of clinical significance in situations where the immune system is 'overdamped'. A similar experiment in AIDS patients would be obviously worthwhile, although the underlying pathology is different.

While there is no such profound immunodeficiency disorder in leprosy, there is a precedent for PG-mediated specific immune unresponsiveness in infectious disease. PGs have been implicated in chronic fungal infections, TB, and brucella. ${ }^{3}$ Treatment of mice infected with Schistosoma mansoni with synthetic PGE led to a significant reduction in the development of egg-induced granulomas. ${ }^{15}$ These granulomas result from a $\mathrm{n}$ immune-mediated hypersensitivity response-PGE had no effect on the development of non-immunological foreign body granulomas. Lymphocyte unresponsiveness in $\mathrm{Q}$ fever (caused by the organism Coxiella burneti) has been shown to be mediated by $\mathrm{PGE}_{1}$. Here, antigen-specific $\mathrm{T}$ suppressor cells appear to secrete an unidentified factor enhancing PGE production by monocytes. ${ }^{16}$ Interestingly, antigen-specific $\mathrm{T}$ suppressor activity has also been implicated in leprosy. ${ }^{17}$

\section{Prostaglandins in leprosy}

Leprosy is a spectrum of diseases caused by a single organism. The position in the spectrum, from the lepromatous pole to the tuberculoid pole, depends on the host immune response. In vitro tests of lymphocyte responsiveness mirror clinical and histopathological indicators of the disease state. ${ }^{18}$ The role of immune suppression in determining this response has been studied in some depth, although exactly which cells are involved and how they act is not yet clear.

Nath and colleagues in India have been searching for a monocyte derived suppressor factor; although this has not yet been isolated, its production does not appear to be inhibited by indomethacin, and its physical characteristics make it unlikely to be a PG. ${ }^{19,20}$ Such a factor may act directly to inhibit IL2 production in T cells of lepromatous patients, although alternative (and more complex) models exist. ${ }^{8,21}$ One study ${ }^{22}$ for example, found that the ability of lepromin to induce suppression of in vitro mitogen responsiveness was mediated by two groups of cells, an adherent cell (assumed to be a macrophage) and a non-adherent $\mathrm{T}$ cell.

Other groups have attempted to answer directly the question of whether PGs are involved in the immune response of leprosy. One study ${ }^{23}$ looked at the effect of PG synthetase inhibition on responsiveness of $\mathrm{T}$ cells from normals and leprosy patients from the lepromatous and tuberculoid poles. They found that the unresponsiveness of BL/LL patients was not affected by indomethacin, whereas there was an approximate doubling of in vitro immune reactivity in TT/BT patients. This suggests that PGs are unlikely to be involved in vitro in the hyporesponsiveness of BL/LL patients. Similarly, it also provokes the idea that it might be possible to enhance further the immune responsiveness of $\mathrm{BT} / \mathrm{TT}$ patients using indomethacin. Interestingly, one study ${ }^{3}$ noted that cells become more responsive to PG-induced immunosuppression in the presence of low concentrations of antigen, which would be the case in BT/TT individuals.

One study, ${ }^{24}$ using a guinea-pig $M$. leprae granuloma model and cell sorter found no detectable PGE in the supernatant of cultures of stimulated granuloma cells. This suggests that the demonstrated inability of these granuloma macrophages to act as accessory cells in immune responses ${ }^{25}$ is not due to excess PG production.

Thus in vitro evidence would point against an important role for PGs in the immunosuppression 
of leprosy. While a study of in vivo effects of PG synthetase inhibitors in leprosy has not been found, there may be circumstantial evidence from a study of the effects of other drugs.

\section{Anti-leprosy drugs and prostaglandins}

Drugs involved in the treatment of leprosy may have immunopharmacological effects over and above their antimicrobial action. This may occur either indirectly, through changes in antigen load, or directly via intrinsic immunomodulatory activity. ${ }^{26}$ As it stands, none of these mechanisms have been proven in vivo, but there is some in vitro evidence in the cases of dapsone and clofazimine.

Dapsone has known anti-oxidant properties and may be a weak inhibitor of PG synthesis. ${ }^{27}$ It has been suggested ${ }^{26}$ that it may act as an immunostimulant through its inhibition of autoregulatory PGs, e.g. there may be an increase in lepromin reaction with dapsone therapy. This is currently a matter for debate, for while it is known that reversal reactions, flare-ups of hypersensitivity in patients with borderline disease, may be precipitated by treatment, one study ${ }^{28}$ has provided evidence that dapsone in high doses may have a protective eff ect. However, so little is known about the aetiology of reversal reactions that to draw any conclusions as to the potential role of PGs from these observations is rather difficult.

Clofazimine (or Lamprene), on the other hand, has been claimed to act as an immunosuppressant, inhibiting reversal reactions, and inhibiting in vitro markers of lymphocyte reactivity. ${ }^{29}$ Anderson has suggested that clofazimine and dapsone are mutually antagonistic with respect to PG production, the former having pro-oxidant effect, and therefore enhancing PG production. An interesting example of clofazimine-induced oxidation is the deposition of ceroid, a substance thought to result from lipid peroxidation and condensation, in a patient on such therapy. ${ }^{30}$ While these arguments are attractive, they provide only indirect evidence for a role for PGs in leprosy, since these drugs have many other actions.

\section{Lipids and leprosy}

Another intriguing insight into the potential eff ect of drugs on immunity comes from experiments ${ }^{31}$ with Chaulmoogra and other oils. Chaulmoogra oil had been used for many years in the treatment of leprosy, taken 'internally, externally and eternally'. Its mode of action is unknown, but is thought to be due to the action of fatty acids of high melting point (hydnocarpic, and chaulmoogric). His account of the effect of such oil on a patient is interesting: 'There was . . . thickening of the tissues, febrile reaction ... swelling up and sof tening with subsequent more rapid resorption of the lesions.'

This sounds very much like an 'upgrading reversal reaction' using modern terminology. This could, of course, be explained through a release of antigen as a result of antimicrobial activity, but Rogers then went on to perform similar experiments with fish oils (cod-liver oil). He reports 51 cases treated with cod-liver oil alone, of whom $80 \%$ improved or cleared, and $65 \%$ cleared completely over the course of 1 year. Rogers notes that the oil appears to have no direct effect on the mycobacteria themselves.

How might these oils be acting? I propose that it may be via an inhibitory action on PG synthesis. It is known that eicosapentenoic acid (EPA) found in fish oils inhibits the synthesis of and is poorly synthesized to PGs. ${ }^{32,33}$ Indeed, increase of EPA in the diet may lead to an increase in mitogen-induced lymphocyte responsiveness via PG depletion. EPA derived from fish oil is one example of an ' $n-3$ ' fatty acid - and it is suggested that all lipids in this group may be inhibitors of PG synthesis. ${ }^{34}$ Linolenic acid, for example, may have a 'restraining' effect on PGE metabolism. ${ }^{35}$

Inhibition of PG synthesis by EPA in fish oil may cause immunostimulation, therefore through release of suppressor activity and promote resolution of leprosy lesions. Hydnocarpic acid itself may also have a weak antioxidant effect by virtue of its resonance hybrid forms (Carpenter K., pers. 
comm.). It would be of great interest to know the effects of this and other oils on PG synthesis in normals and leprosy patients. The original experiments may be over 60 years old, but we may still have something to learn from them.

\section{Prostaglandins in leprosy: a synthesis}

There is good evidence that PGs are involved in regulation of the normal immune response, and that they may play a part in various disease states. There is little evidence as yet, however, that they are important in leprosy. This question is not purely academic. We have cheap and reliable antiprostaglandin agents available which could be of use in this disease. I would like to consider two possible situations.

1 PGs are relevant to the immune defect in leprosy. In this case, there is a chance that PG synthetase inhibitors may accelerate a cure in concert with current antileprosy drugs. This may be restricted to certain areas of the leprosy spectrum, or certain stages of the disease. The effect on reversal reactions is also unpredictable, since we do not understand their basis. Immunotherapy for leprosy of this kind is not such a far-fetched idea, for this is effectively what is being attempted with the BCG vaccine.

2 PGs are irrelevant to the immune def ect in leprosy. In this case, our hopes of immunomodulation with simple drugs such as aspirin are dashed. There may, however, be an important role for antiprostaglandins as anti-inflammatory agents. In reversal reactions, this is a sudden flare-up of hypersensitivity, as discussed, and this is accompanied by oedema formation within the lesions. Such oedema within a nerve sheath may lead to irreversible ischaemic damage. Currently, emergency treatment for these reactions relies on steroids, which are powerf ul but dangerous drugs, particularly in the hands of those with little experience in their use. Simple anti-inflammatory agents may be very usef ul in these situations to counteract the critical oedema, and if indeed it does turn out that these drugs have few immunostimulant properties (which might provoke or potentiate the reaction), a trial of their use may be warranted.

A scheme for the further investigation of the role of PGs in leprosy is set out below:

EXPT. 1 Effect of aspirin/indomethacin on skin test responsiveness

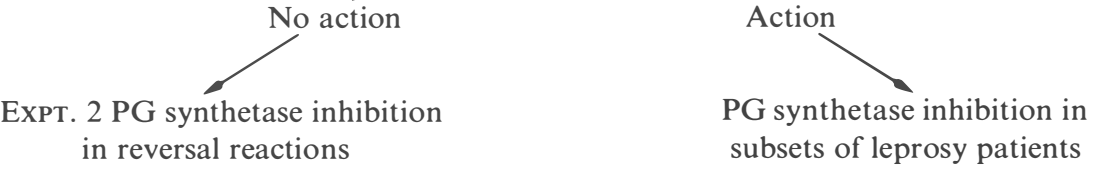

PGs may be relevant or irrelevant to leprosy. In either case, PG synthetase inhibitors may have a role in the future therapy of the disease. The drugs are cheap, and while the side-effects (notably gastrointestinal bleeding) are not insignificant, they compare favourably to those of steroids. Essentially, there is little to lose by trying - and a great deal to gain. In this essay I hope to have demonstrated that we have at least a rational basis for such an attempt.

\section{Acknowledgments}

I would like to thank Dr A C McDougall for his help and encouragement with this essay, Sally Cowley from the Royal College of Surgeons for pointing me in the right direction, and Dr J S Goodwin for providing me with some excellent reading material. 


\section{References}

1 Wardle EN. Guide to the Prostaglandins. Br J Hosp Med, 1985; 34: 229-32.

2 Tremoli E, Maderna P, Eynard A. In vitro effects of aspirin and NSAID's on the formation of 12hydroxyeicosa pentenoic acid by platelets. Prostagl Leukotr Med, 1986; 23: 117-22.

3 Goodwin JS, Webb DR. Regulation of the immune response by prostaglandins. Clin Imm Immuno path, 1980; 15: $106-22$.

4 Parnham MJ, Schoester GA, Van der Kwast TH et al. Enhancement by PGE, and essential fatty acid deficiency of the passive transfer of delayed hypersensitivity to PPD in rats. Comparison with effects on delayed hypersensitivity to SRBC in mice. Int J Immunopharmacol, 1979; 1: 119-29.

5 Muscoplat CC, Rakich PM, Thoen CO et al. Enhancement of lymphocyte blastogenic and delayed hypersensitivity skin response by Indomethacin. Inf Immun, 1978; 20: 627-31.

6 Goodwin JS, Selinger DS, Meissner RP et al. Effect of Indomethacin in vivo on humoral and cell immunity in humans. Inf Immun, 1978; 19: 430-3.

7 Kurland JL, Blockman R. PGE production by human blood monocytes and morse peritoneal macrophages. $J$ exp Med, 1979; 147: 952-7.

${ }^{8}$ Honda M, Steinberg AD. Effects of PGE2 on the response of T cell subsets to mitogen and autologous non T cell stimulation. Clin Imm Immunopath, 1984; 33: 111-22.

${ }^{9}$ Plaut M. Lymphocyte hormone receptors. Ann Rev Immun, 1987; 5: 621-69.

10 Gualde N, Aldigier JC, Mexnan S et al. Regulation of the immune response by eicosanoid acids. In Prostaglandins and Immunity. (ed.) Goodwin JS (ed.), Publ Boston: Martinus Nijhoff 1985; pp.147-60.

11 Goodwin JS, Messner RP, Bankhurst AD et al. Prostaglandin-producing suppressor cells in Hodgkin's disease. NEUM, 1977; 297: 963-8.

12 Lynch NR, Salomon JC. Tumour growth inhibition and potentiation of immunotherapy by Indomethacin in mice. J Nat Canc Inst, 1979; 62: 117-21.

13 Goodwin JS, Webb DR. Partial reversal of cellular immune defect in common variable immunodeficiency with Indomethacin. J Clin Lab Imm, 1978; 1: 197-9.

15 Fantone JC, Kunkel SL, Zurier RB. Effects of prostaglandins on invivoimmune and inflammatory reactions. In Prostaglandins and Immunity. Goodwin JS (ed.), Publ Boston: Martinus Nijhoff 1985; pp.123-46.

${ }^{16}$ Koster FT, Williams JC, Goodwin JS. Cell immunity in Q fever; specific unresponsiveness in Q fever endocarditis. J Inf Dis, 1985; 152: 1283-9.

17 Strickland NH. Influence of immunosuppression and immunodeficiency on infection with leprosy and TB. Int J Lepr, 1985; 53: 86-100.

18 Myrvang G, Godal T, Ridley DS et al. Immune response to $M$. leprae and other mycobacterial antigens throughout the clinical and histopathological spectrum of leprosy. Clin exp Imm, 1973; 14: 541-83.

19 Salgame PR, Mahaderan PR, Antia NH. Mechanism of immunosuppression in leprosy; presence of suppression factor(s) from macrophages of leprosy patients. Inf Immun, 1983; 40: 1119-26.

20 Sathish M, Bhutani LK, Sharma AK et al. Monocyte-derived soluble suppressor factors in patients with lepromatous leprosy. Inf Immun, 1983; 42: 890-9.

21 Nath I, Jayaraman J, Sathish M. Inhibiting of 1 L-2 production by adherent cell factors from lepromatous leprosy patients. Clin exp Imm, 1984; 58: 531-8.

22 Mehra V, Mason LH, Fields JP et al. Lepromin induced suppressor cells in patients with leprosy. J Immunol, 1979; 4: 1813-7.

23 Bahr GM, Rook GAW, Stanford JL. Prostaglandin-dependent regulation of in vitro proliferative response to mycobacterial antigens of peripheral blood lymphocytes from normal donors and f rom patients with TB or leprosy. Clin exp Imm, 1981; 45: 646-53.

${ }^{24}$ Montreewasuwat N, Curtis J, Turk JL. 1L-7 and prostaglandin production by cells of the mononuclear phagocyte system isolated from mycobacterial granulomas. Cell Immunol, 1987; 104: 12-23.

${ }^{25}$ Gupta SK, Curtis V, Turk VL. Accessory Cell Function of Cells of the Mononuclear Phagocyte System Isolated from Mycobacterial Granulomas. Cell Immunol. 1985; 91: 42-45.

26 Anderson R. Enhancement by Clofazimine and inhibition by Dapsone of production of PGE2 by human polymorphonuclear leukocytes in vitro. Antimicrob Ay Chemother, 1985; 27: 257-62.

27 Ramu G, Sengupta J, Desikan KJ. Influence of sulphone therapy on lepromin reaction. Lepr Rev, 1980; 51: 207-14.

28 Barnetson RS, Pearson JM, Rees RJ. Evidence of prevention of borderline leprosy reactions by Dapsone. Lancet, 1976; ii: 1171-2.

29 Van Rensburg CE, Gatner EM, Im Kamp FM et al. Effects of Clofazime alone or combined with Dapsone on neutrophil and lymphocyte function in patients with lepromatous leprosy and normal individuals. Antimicrob Ag Chemother, 1982; 21: 693-7.

${ }^{30} \mathrm{McDougall}$ AC, Jones RL. Intra neural acid-like pigment following the treatment of lepromatous leprosy with clofazimine (B663: Lamprene). J Neurol neurosurg psych, 1981; 44: 116-20. 
31 Rogers Sir L. Chaulmoogra oil in leprosy and TB. The successf ul treatment of leprosy by injection of the fatty acids of chaulmoogra and other oils, and its bearing on the TB problem. Lancet, 1921; i: 1178-90.

32 Pickett WC, Nytko D, Dondero Z et al. The effect of endogenous eicosapentaenoic acid on Polymorphonuclear leukocytes and PAF biosynthesis. Prostagl Leukotr Med, 1986; 23: 135-40.

33 Higgs GA. The effect of dietary intake of fatty acids on prostaglandin and leukotriene synthesis. Proc. Nutr. Soc. $1985 ;$ 44: 181.

${ }^{34}$ Culp BR, Titus B G et al. Inhib of Prostaglandin biosynthesis by eicosapentaenoic acid. Prostagl in Med, 1979; 3: 269-78.

35 Budowsk P, Crawf ord MA. Linolenic acid as a regulator of the metabolism of arachidonic acid deficiency: implications of the ratio n-6:n-3 fatty acids. Proc Nutr Soc, 1985; 49: 221-9. 